\title{
LEDs for Fluorescence Microscopy
}

\author{
I.T. Young*, Y. Garini, H.R.C. Dietrich, W. van Oel, G. Liqui Lung \\ Quantitative Imaging Group, Department of Imaging Science \& Technology, Faculty of Applied \\ Sciences, Delft University of Technology, Lorentzweg 1, NL-2628 CJ Delft, The Netherlands
}

\begin{abstract}
Traditional light sources for fluorescence microscopy have been mercury lamps, xenon lamps, and lasers. These sources have been essential in the development of fluorescence microscopy but each can have serious disadvantages: lack of near monochromaticity, heat generation, cost, lifetime of the light source, and possible distortions due to coherence effects.

We are examining the possibility of using the new high-power light-emitting diode (LED) sources as alternatives to the above mentioned sources. LED sources are near monochromatic, are inexpensive, produce little heat, have no coherence problems, have extended lifetimes, are small, and can easily be modulated.

We describe experiments comparing various LEDs to other light sources. We compare, for example, a $530 \mathrm{~nm}$ LED to the $546 \mathrm{~nm}$ line from a mercury lamp on a fluorophore whose absorption maximum is broad and in the middle between these two wavelengths.
\end{abstract}

Keywords: high-power light-emitting diodes, fluorescence microscopy, multi-spectral sources, quantitative microscopy

\section{INTRODUCTION}

Long before there were incandescent lamps, quartz halogen lamps, mercury lamps, xenon lamps, and lasers, microscopes were used to examine specimens using sunlight, oil lamps, or candlelight reflected by a mirror and brought to the specimen plane by a condenser. More than 300 years ago John Marshall, for example, manufactured such instruments in London ${ }^{1}$. These instruments made it possible to study specimens by transmitted and reflected light but it remained until the early $20^{\text {th }}$ century before a range of new microscope imaging modalities were developed and exploited. Köhler is reported to have observed fluorescence as early as 1904 but it was not until the work of Ploem ${ }^{2}$ in the 1960's that fluorescence microscopy became a practical tool. The significance of his epi-illumination architecture was immediately recognized and the importance of fluorescence as a probe for biological structure was demonstrated shortly thereafter by the work of Caspersson et $\mathrm{al}^{3,4}$ in their use of fluorescent staining techniques to reveal bands in metaphase chromosomes.

For the past 30 years, developments in fluorescence microscope instrumentation have proceeded along four main lines:

1) Opticaldevelopments - The goal of this work has been to produce optical images with improved contrast, fewer aberrations, and - in particular-improved spatial resolution. This work has led, for example, to the optical techniques known as confocal microscopy, two-photon microscopy, and stimulated emission depletion (STED) microscopy which currently holds a resolution record of $33 \mathrm{~nm}^{5,6}$ which is $\lambda / 23$.

2) Sensor developments - While the earliest work in the analysis of fluorescent images used either film or photomultipliers as the sensing "agent", modern systems can make use of a range of available detectors. These include cooled, ultra-sensitive, high-speed CCD cameras; avalanche photodiode arrays; compact, ultra-sensitive photomultipliers; and inexpensive, high-quality color video cameras.

3) Computer developments - The developments in both hardware and software have made it possible to acquire, store, process, and analyze microscope images that can be characterized by three spatial dimensions, dynamic changes, and multi-spectral characterizations for every point in an object's spatial volume. It is the advances in the use of computers in microscope imaging-quantitative microscopy - that have made the microscope a tool for $\mathrm{N}$-dimensional imaging.

\footnotetext{
young@ph.tn.tudelft.nl; phone +31-15-278-1416; fax +31-15-278-6740; http://www.ph.tn.tudelft.nl/
} 
4) Illumination developments - The filters that can now be employed to distinguish one or more emission wavelengths from one or more excitation wavelengths have improved dramatically. Multi-bandpass filters can now be standard components of a fluorescence microscope system. Further, the illumination of the specimen has also improved dramatically first with the improvements and standardizations of excitation sources such as high-pressure mercury and xenon gas-discharge lamps, the introduction of quartz halogen lamps, and the laser.

It is this last area-the development of improved light sources for fluorescence microscopy-that is the focus of this paper.

\section{BACKGROUND}

The light sources that are currently used in fluorescent microscopy are high-pressure mercury lamps, high-pressure xenon lamps, or lasers. The wavelengths of excitation light provided by each of these sources as well as other relevant information are given in Table 1.

Table 1: Common light sources for fluorescence microscopy

\begin{tabular}{|c|c|c|c|c|}
\hline Light Source & Wavelengths $[\mathrm{nm}]$ & Power & Price & Lifetime (hours) \\
\hline Mercury (HBO) lamp & $\begin{array}{c}\text { peaks: } 313,334,365, \\
406,435,546,578\end{array}$ & $100[\mathrm{~W}]$ & $\approx \$ 170$ & $\approx 200$ \\
\hline Xenon (XBO) lamp & $\begin{array}{c}\text { "uniform”: } \\
400 \leq \lambda \leq 800\end{array}$ & $75[\mathrm{~W}]$ & $\approx \$ 200$ & $\approx 400$ \\
\hline Argon ion laser & $\begin{array}{c}\text { lines: } 351,364,457, \\
476,488,514\end{array}$ & $50-100[\mathrm{~mW}]$ & $>\$ 5000$ & $\approx 6000$ \\
\hline $\begin{array}{c}\text { Helium-Neon (HeNe) } \\
\text { laser }\end{array}$ & lines: $543,594,633$ & $1.5-10[\mathrm{~mW}]$ & $>\$ 1500$ & $\approx 40,000$ \\
\hline
\end{tabular}

The specific advantages and disadvantages associated with each type of source are given in Table 2 .

Table 2: Advantages and disadvantages of common light sources

\begin{tabular}{|lll|}
\hline Mercury (HBO) lamp & Advantages: & $\begin{array}{l}\text { multiple spectral peaks, incoherent source, reasonable } \\
\text { price, compact light emitting surface }\end{array}$ \\
\cline { 3 - 3 } & Disadvantages: & $\begin{array}{l}\text { excessive heat production, short lifetime, ozone } \\
\text { production, excitation and emission filters required, } \\
\text { difficult to modulate }\end{array}$ \\
\hline Xenon (XBO) lamp & Advantages: & $\begin{array}{l}\text { broadband spectrum, incoherent source, reasonable } \\
\text { price, compact light emitting surface }\end{array}$ \\
\hline Disadvantages: & $\begin{array}{l}\text { excessive heat production, short lifetime, ozone } \\
\text { production, excitation and emission filters required, } \\
\text { difficult to modulate }\end{array}$ \\
\hline
\end{tabular}




\begin{tabular}{|c|c|c|}
\hline \multirow[t]{2}{*}{ Lasers } & Advantages: & $\begin{array}{l}\text { monochromatic spectral lines, high power per spectral } \\
\text { line, relatively long lifetime, compact light emitting } \\
\text { surface }\end{array}$ \\
\hline & Disadvantages: & $\begin{array}{l}\text { excessive heat production, high price, coherent } \\
\text { source, emission filters required, high voltage } \\
\text { required, difficult to modulate }\end{array}$ \\
\hline
\end{tabular}

This inventory of properties then brings us to the following question. If we were asked to describe an ideal light source for fluorescence microscopy, what would it be like? The advantages and disadvantages listed above suggest that the characteristics would be:

- near monochromatic source

- multiple wavelengths

- reasonable power per wavelength

- incoherent source

- compact light emitting surface

- $\quad$ easily modulated
- $\quad$ negligible heat production

- no ozone production

- no excitation filter needed

- low price

- $\quad$ long lifetime

- low-voltage operation

While it is common in the investigation of improved technologies to produce a "wish" list of properties, it is rarely the case that all of our wishes can be fulfilled; we are usually forced to accept compromises. With the development of the latest generation of high-power LED light sources, however, the characteristics of these sources are so similar to our desired goals that we can anticipate a new generation of light sources for fluorescence microscopy that meet all of our requirements.

\section{LED SOURCES}

The past ten years have seen the development of a range of LEDs that is capable of producing a significant amount of luminous flux (or radiant power). Within the past five years the spectral range of the commercially-available LEDs has expanded in the visible range from $460 \mathrm{~nm}$ to $645 \mathrm{~nm}^{1}$. The acceptance of these devices have been extraordinary with applications ranging from traffic signals to indoor and outdoor commercial signs. More than 200,000,000 LEDs are being shipped each month. What this means is that, while the domain of fluorescence microscopy can never aspire to be a significant market for these developments, the development costs for new generations of LEDs will be borne by others and the fluorescence microscopy can "ride this wave."

The characteristics of these LEDs that make them so appealing are given in Tables 3 and 4.

Table 3: General Characteristics of high-power LEDs

\begin{tabular}{|c|c|c|c|c|}
\hline Light Source & Modulation Bandwidth & Power input & Price & Lifetime (hours) \\
\hline \multirow{2}{*}{ High-Power LEDs } & $>1 \mathrm{MHz}$ & $1[\mathrm{~W}]$ & $\approx \$ 12$ & $>50,000$ \\
& & $5[\mathrm{~W}]$ & $\approx \$ 42$ & $>$ \\
\hline
\end{tabular}

\footnotetext{
${ }^{1}$ All LED specifications refer to Luxeon Emitter LEDs, Lumileds Lighting, 370 West Trimble Road, San Jose, California, http://www.lumileds.com/.
} 
Table 4: Spectral Characteristics of high-power LEDs

\begin{tabular}{|c|c|c|c|}
\hline Wavelength [nm] & Flux & Color & $\begin{array}{c}\text { Spectral Width } \\
\Delta \lambda[\mathrm{nm}]\end{array}$ \\
\hline $\begin{array}{c}455 \\
\text { (model \# LXHL-PR03) }\end{array}$ & $\begin{array}{c}100[\mathrm{~mW}] \\
500[\mathrm{~mW}]\end{array}$ & "Royal" Blue & $\approx 15$ \\
\hline $\begin{array}{c}470 \\
\text { (model \# LXHL-PB01) }\end{array}$ & $\begin{array}{c}5[\mathrm{~lm}] \\
30[\mathrm{~lm}]\end{array}$ & Blue & $\approx 12$ \\
\hline $\begin{array}{c}505 \\
\text { (model \# LXHL-PE01) }\end{array}$ & $\begin{array}{c}30[\mathrm{~lm}] \\
120[\mathrm{~lm}]\end{array}$ & Cyan & $\approx 18$ \\
\hline $\begin{array}{c}530 \\
\text { (model \# LXHL-PM01) }\end{array}$ & $\begin{array}{c}25[\mathrm{~lm}] \\
120[\mathrm{~lm}]\end{array}$ & Green & $\approx 23$ \\
\hline $\begin{array}{c}\text { 590 } \\
\text { (model \# LXHL-PL01) }\end{array}$ & $36[\mathrm{~lm}]$ & Amber & $\approx 12$ \\
\hline $\begin{array}{c}\text { 617 } \\
\text { (model \# LXHL-PH01) }\end{array}$ & $55[\mathrm{~lm}]$ & Red-Orange & $\approx 12$ \\
\hline $\begin{array}{c}625 \\
\text { (model \# LXHL-PD01) }\end{array}$ & $44[\mathrm{~lm}]$ & Red & $\approx 12$ \\
\hline
\end{tabular}

The spectral shape of each of these standard seven emitters is approximately Gaussian and shown in Figure 1. There is a possibility that other emitters could become available with a wavelength spacing of $5 \mathrm{~nm}$, that is, a central emission wavelength spaced every $5 \mathrm{~nm}$ across the visible spectrum, but these are not currently available ${ }^{7}$.

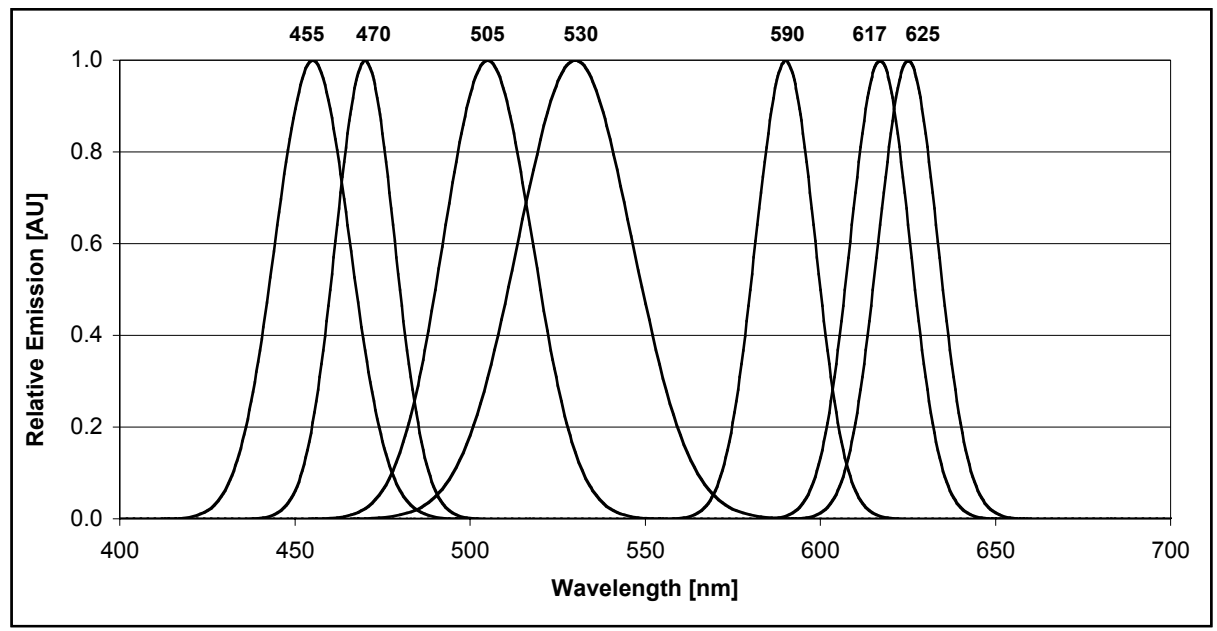

Figure 1: Emission characteristics of various LEDs

An important aspect for determining how efficient a light source can be in the context of microscopy is the compactness of the actual light emitter. In gas discharge lamps this is the size of the arc and in the lasers the size of the beam at the exit coupler mirror. The approximate values for each of the sources described above are given in Table 5. 
Table 5: Size of emitter

\begin{tabular}{|c|c|c|c|c|}
\cline { 2 - 5 } \multicolumn{1}{c|}{} & Mercury lamp & Xenon lamp & Lasers & LEDs \\
\hline Size & $\begin{array}{c}0.25 \times 0.25 \\
{\left[\mathrm{~mm}^{2}\right]}\end{array}$ & $\begin{array}{c}\approx 0.25 \times 0.50 \\
{\left[\mathrm{~mm}^{2}\right]}\end{array}$ & $\varnothing \approx 1 \mathrm{~mm}$ & $\begin{array}{c}0.5 \times 0.5 \\
{\left[\mathrm{~mm}^{2}\right]}\end{array}$ \\
\hline
\end{tabular}

The use of one or more LEDs allows us to meet the demands presented above. The need for a multi-spectral source could be met by optically combining the outputs of various LEDs. The non-overlapping spectra could then be switched on and off as desired.

The choice of fluorescent dyes presents an interesting challenge. As long as we are restricted to the seven wavelengths listed above, then we must look for fluorescent dyes that match our wavelengths as opposed to light sources that match our fluorophores. This should not be an insurmountable problem as shown in Table 6.

Table 6: Fluorophores that can be used with LEDs

\begin{tabular}{|c|c|c|}
\hline Wavelength [nm] & LED Color & Commercially available dye $e^{8,9}$ \\
\hline $455 \pm 10$ & "Royal" Blue & $\begin{array}{l}\text { SYTOX, blue SYTO blue, DiA, Acridine orange (RNA), } \\
\text { BOBO-1, BO-PRO-1, NBD amines, CTC Formazan, } \\
\text { Chromomycin A3, CFP, CFP/YFP FRET }\end{array}$ \\
\hline $470 \pm 10$ & Blue & $\begin{array}{l}\text { Acridine orange (RNA), BOBO-1, BO-PRO-1, NBD amines, } \\
\text { Di-8-ANEPPS, Di-4-ANEPPS, Fura Red, FM 1-43, GFP } \\
\text { Wild Type (non-UV Excitation), GFP/DsRed FRET }\end{array}$ \\
\hline $505 \pm 10$ & Cyan & $\begin{array}{l}\text { Alexa Fluor 488, Oregon green, Rhodamine 110, JC-1, } \\
\text { Acridine orange (DNA), NeuroTrace, RH 414, Rhodamine } \\
\text { green, Alexa Fluor 500, BCEDF (high pH), LysoTracker } \\
\text { green, SYTOX green B, BODIPY FL, Calcium green, Fluo- } \\
3 \text {, FM 4-64, JC-9, Magnesium green, Rhodamine 123, } \\
\text { Sodium green, TOTO-1, TO-PRO-1 B, SYTO green, } \\
\text { SpectrumGreen \#2, YFP, DM-NERF (4.5-6.5 pH), Cl- } \\
\text { NERF (low pH) }\end{array}$ \\
\hline $530 \pm 10$ & Green & $\begin{array}{l}\text { Eosin, 6-Carboxyrhodamine 6G, 6-JOE, EthD-1 } 3 \text {, Alexa } \\
\text { Fluor 532, Propidium iodide } \beta, \text { SYTO orange, SNAFL-2, } \\
\text { LysoTracker yellow, JOJO-1, JO-PRO-1 }\end{array}$ \\
\hline $590 \pm 10$ & Amber & $\begin{array}{l}\text { ROX, X-rhod-1, BODIPY TR-X, Alexa Fluor 594, Calcium } \\
\text { crimson, JC-1, Texas red, Red-X, SpectrumRed, Cy3.5, 5- } \\
\text { Carboxynaphthofluoroscein }\end{array}$ \\
\hline $617 \pm 10$ & Red-Orange & $\begin{array}{l}\text { YOYO-3, YO-PRO-3 B, SYTO red, C-phycocyanin, R- } \\
\text { phycocyanin }\end{array}$ \\
\hline $625 \pm 10$ & Red & $\begin{array}{l}\text { SYTO red, BODIPY 630/650, Alexa Fluor 633, C- } \\
\text { phycocyanin, R-phycocyanin }\end{array}$ \\
\hline
\end{tabular}




\section{PRELIMINARY EXPERIMENTS}

We have performed a first set of experiments to evaluate the use of high-power LEDs. We constructed a housing for eight different LEDs, the seven colored LEDs discussed above as well as a white LED (model \#LXHL-PW01). The housing was designed to fit on a Leica TCS_SP2 AOBS confocal microscope at the site where the 100W mercury or xenon lamp is usually attached to the microscope body. This is shown in Figure 2.

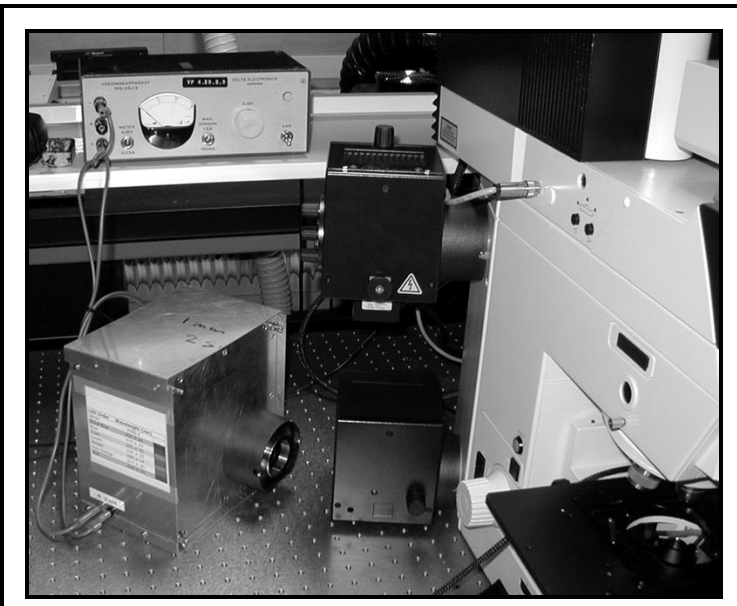

Figure 2: (Left-Top) The LED light source is to be mounted on the left side of the confocal microscope body. It is below and to the left of the mounted mercury lamp housing. (Left-Bottom) Close-up showing mounting flange and several of the LEDs. (Right-Bottom) Detail of the interior of the LED housing. A total of 10 LEDs have been mounted on a disk permitting rotation to the chosen wavelength. Not shown are the mechanical adjustment controls for correct positioning of the LED.
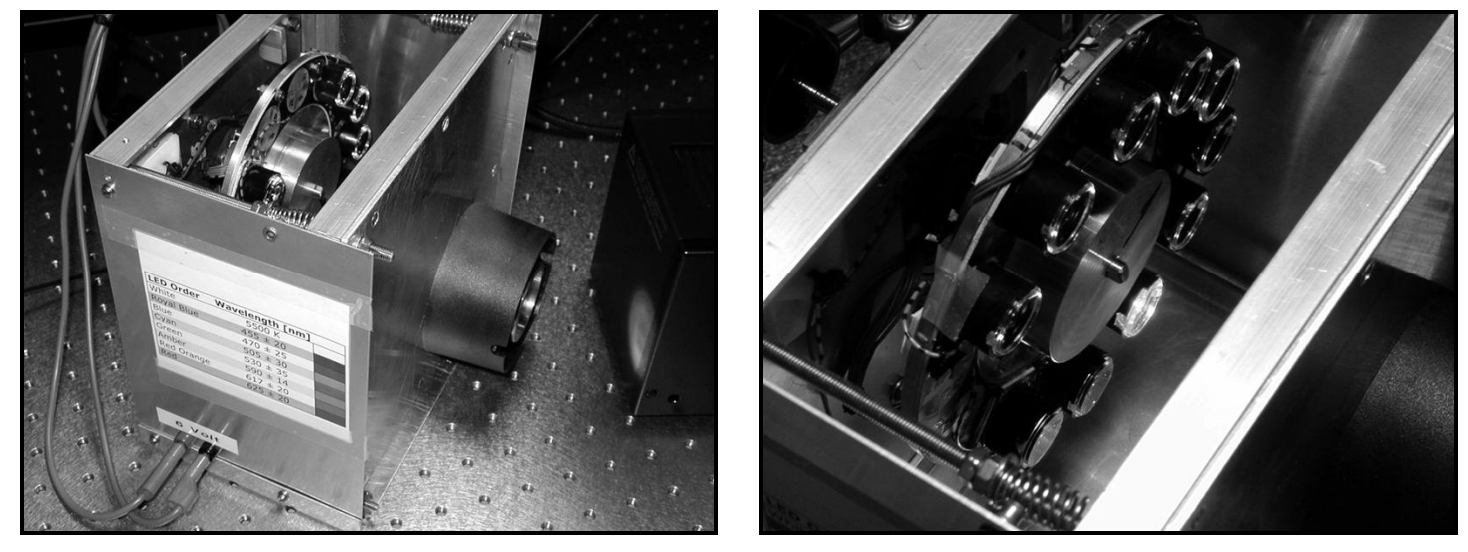

\subsection{Radiometric experiments}

In the first set of experiments we examined the radiometric power produced at the specimen plane of the microscope for both a $100 \mathrm{~W}$ mercury (HBO) lamp and four different LEDs using a 20x lens with a NA of 0.4. The LEDs were operated at a $600 \mathrm{~mW}$ input level or $60 \%$ of the maximum rated input power. Two "blue" LEDs (455 nm and $470 \mathrm{~nm}$ ) were used with an I3 filter block. The I3 filter block has an excitation bandpass filter from $450 \mathrm{~nm}$ to $490 \mathrm{~nm}$, a dichroic mirror at $510 \mathrm{~nm}$, and a longpass filter of $515 \mathrm{~nm}$. The two other LEDs $(505 \mathrm{~nm}$ and $530 \mathrm{~nm})$ were used with an N2.1 filter block. The N2.1 filter block has an excitation bandpass filter from $515 \mathrm{~nm}$ to $560 \mathrm{~nm}$, a dichroic mirror at $580 \mathrm{~nm}$, and a longpass filter of $590 \mathrm{~nm}$. Radiometric measurements were made using an Ophir radiometer (Ophir Optronics, Danvers, Massachusetts). Results of the measurements are given in Table 7 where, in the last column, we correct for having used only $60 \%$ of the maximum rated input power to the LEDs. 
Table 7: Radiometric power of LEDs vs. mercury lamp

\begin{tabular}{|c|c|c|c|c|c|}
\hline LED [nm] & LED Power $[\mathrm{mW}]$ & Hg Power $[\mathrm{mW}]$ & Filter Block & Hg / LED & corr. ratio \\
\hline 455 & 0.18 & 4.52 & $\mathrm{I} 3$ & 25 & $\mathbf{1 5}$ \\
\hline 470 & 0.16 & 4.15 & $\mathrm{I} 3$ & 26 & $\mathbf{1 6}$ \\
\hline 505 & 0.04 & 11.34 & $\mathrm{~N} 2.1$ & 284 & $\mathbf{1 7 0}$ \\
\hline 530 & 0.02 & 10.00 & $\mathrm{~N} 2.1$ & 500 & $\mathbf{3 0 0}$ \\
\hline
\end{tabular}

It is clear that our LED housing is not performing as it should. Too little light is reaching the microscope objective. It is not surprising that the $505 \mathrm{~nm}$ (Cyan) is performing so poorly; its peak wavelength is outside the range of the excitation bandpass filter. But no such simple explanation is available for the results at $530 \mathrm{~nm}$ (Green). The results for $455 \mathrm{~nm}$ (Royal Blue) and $470 \mathrm{~nm}$ (Blue) are disappointing but 10x to 20x better than the other two wavelength results.

\subsection{Fluorescence experiments}

In a second set of experiments we looked at Rhodamine B fluorescence using the nanowell technology that we have developed $^{10}$. Rhodamine B has an absorption peak located at approximately $558 \mathrm{~nm}$ and the two light sources-the 530 $\mathrm{nm}$ LED and the $546 \mathrm{~nm}$ line of the mercury lamp-are, respectively, 46\% and 79\% absorbed. Again, the LED was operated at a $600 \mathrm{~mW}$ input level which is $60 \%$ of its rated maximum.

We used a $4 \mathrm{x}$ lens with an NA of 0.1 for both the $100 \mathrm{~W}$ mercury (HBO) lamp and the $530 \mathrm{~nm}$ LED and the N2.1 filter block. A dye concentration of $1.0 \mathrm{mg} / \mathrm{ml}$ was used and deposited in our nanowells, each well having a capacity of about $1 \mathrm{nl}$. Measurements were made with a Hamamatsu Orca-ER camera (model C4742) with a maximum possible signal of 4095 and in this experiment a background level of 200. Different exposure times were used for the LED source and the mercury source. The results of these measurements are shown in Table 8.

Table 8: Fluorescence measurement of Rhodamine B: LEDs vs. mercury lamp

\begin{tabular}{|r|ccc|ccc|}
\hline exposure $(m s)$ & \multicolumn{3}{|c|}{$\mathbf{1 0 0 0}$} & \multicolumn{3}{c|}{$\mathbf{0}$} \\
\cline { 2 - 7 } & LED well & LED bkg. & Net LED signal & Hg well & Hg bkg. & Net Hg signal \\
Well \#1 & 823 & 221 & 602 & 2310 & 259 & 2051 \\
Well \#2 & 836 & 226 & 610 & 2205 & 238 & 1967 \\
Well \#3 & 828 & 227 & 601 & 2459 & 245 & 2214 \\
\hline Average & $\mathbf{8 2 9}$ & $\mathbf{2 2 5}$ & $\mathbf{6 0 4}$ & $\mathbf{2 3 2 5}$ & $\mathbf{2 4 7}$ & $\mathbf{2 0 7 7}$ \\
\hline
\end{tabular}

Normalizing these measurements for integration time gives a Net Signal per ms of 0.604 for the LED and 207.7 for the $\mathrm{Hg}$ lamp. The ratio $\mathrm{Hg} / \mathrm{LED}=207.7$ / $0.604=344$ must be normalized for LED input power and the relative absorption values giving a normalized value of norm. $H g / L E D=344 * 0.6 *(0.46 / 0.79)=\mathbf{1 2 0}$. This again shows that we have not yet been able to deliver all of the luminous flux from the LED source to the object plane of the microscope objective lens.

\section{SUMMARY AND CONCLUSIONS}

The results of these experiments should not deter us from concluding that LEDs offer a significant opportunity to improve the light sources used in fluorescence microscopy. Microscope manufacturers have had some 70 years to develop and improve episcopic illumination for fluorescence microscopy ${ }^{11}$. We will continue our development of a multi-spectral LED microscopy light source. We expect that the major microscope manufacturers will show interest in this as well. 
No discussion of fluorescence microscopy would be complete without at least one illustration of fluorescent images. In Figure 3 we show two images of SpectrumGreen-stained metaphase chromosomes, one taken with the mercury lamp and one with the $470 \mathrm{~nm}$ (Blue) LED. In both cases a 100x oil-immersion objective with an NA = 1.4 and the I3 filter cube were used.

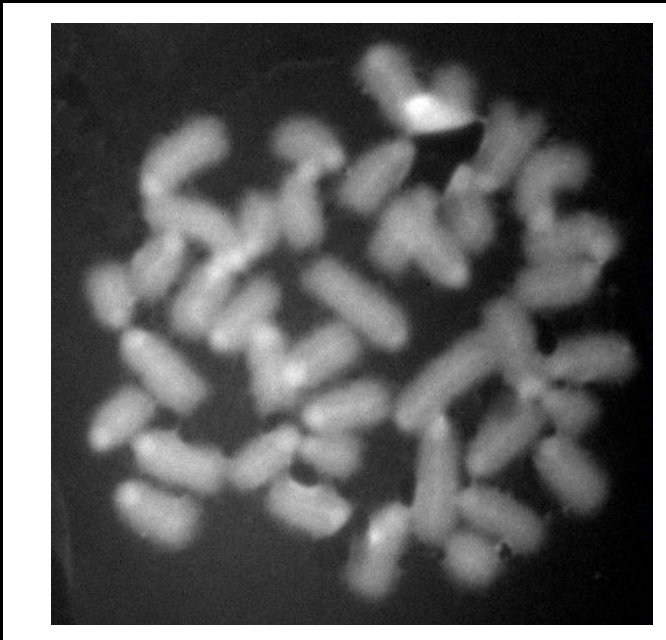

Figure 3a: Metaphase chromosomes illuminated with the mercury $(\mathrm{Hg})$ lamp and with an exposure (integration) time of $0.75 \mathrm{~s}$.

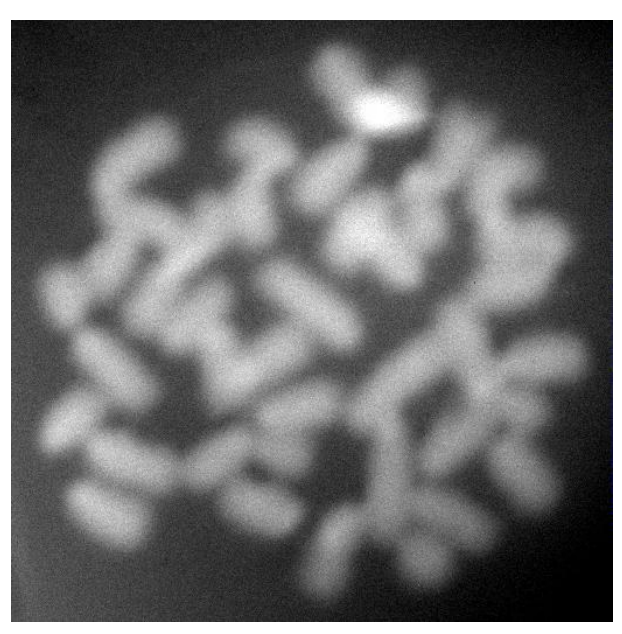

Figure 3b: Metaphase chromosomes illuminated with the $470 \mathrm{~nm}$ (Blue) LED and with an exposure (integration) time of $5.00 \mathrm{~s}$.

Although the I3 filter cube is not the optimum choice for the SpectrumGreen fluorophore, for purposes of comparison it is adequate.

\section{ACKNOWLEDGMENTS}

This work was partially supported by the Physics for Technology program of the Foundation for Fundamental Research in Matter (FOM) and the Delft Inter-Faculty Research Center LifeTech (DIOC-LifeTech).

\section{REFERENCES}

${ }^{1}$ H.R. Purtle, History of the Microscope, Armed Forces Institute of Pathology, Washington, DC, 1974.

${ }^{2}$ J.S. Ploem, "The use of a vertical illuminator with interchangeable dichroic mirrors for fluorescence microscopy with incident light," Zeitschrift für wissenschaftliche Mikroscopie, 68, pp. 129-142, 1967.

${ }^{3}$ T. Caspersson, L. Zech, and C. Johansson, "Differential banding of alkylating fluorochromes in human chromosomes," Experimental Cell Research, 60, pp. 315-319, 1970.

${ }^{4}$ T. Caspersson, L. Zech, C. Johansson et al., "Identification of human chromosomes by DNA-binding fluorescent agents," Chromosoma, 30, pp. 215, 1970.

${ }^{5}$ M. Dyba and S.W. Hell, "Focal Spots of Size $\lambda / 23$ Open Up Far-Field Florescence Microscopy at $33 \mathrm{~nm}$ Axial Resolution," Physical Review Letters, 88, pp. 163901-163904, 2002.

${ }^{6}$ M. Dyba, S. Jakobs, and S.W. Hell, "Immunofluorescence stimulated emission depletion microscopy," Nature Biotechnology, 21:11, pp. 1303-1304, 2003.

${ }^{7}$ A. Perduijn, Private communication: Spectral availability of LEDs, Delft, 1 May 2003.

${ }^{8}$ Glen Spectra, "Filter Sets by Fluorophore: http://www.glenspectra.co.uk/glen/filters/fffluorpn.htm," (2003).

${ }^{9}$ Molecular Probes, "Spectral characteristics and recommended bandpass filter sets for Molecular Probes' dyes: http://www.probes.com/handbook/tables/0372.html," (Haugland, R.P., 2003).

${ }^{10}$ I.T. Young, R. Moerman, L.R. van den Doel et al., "Monitoring enzymatic reactions in nanoliter wells," J. Microscopy, 212:3, pp. 254-263, 2003.

${ }^{11}$ J. Reichman, Handbook of Optical Filters for Fluorescence Microscopy, Chroma Technology Corporation, Brattleboro, Vermont, 2000. 